\title{
Detecting Aquaporin Function and Regulation
}

\author{
Ana Madeira ${ }^{1}$, Teresa F. Moura ${ }^{1,2}$ and Graça Soveral ${ }^{1,3 *}$ \\ ${ }^{1}$ Research Institute for Medicines (iMed. ULisboa), Faculty of Pharmacy, Universidade de Lisboa, Lisboa, Portugal, \\ ${ }^{2}$ Faculdade de Ciências e Tecnologia, Universidade Nova de Lisboa, Caparica, Portugal, ${ }^{3}$ Departamento Bioquimica e \\ Biologia Humana, Faculty of Pharmacy, Universidade de Lisboa, Lisboa, Portugal
}

OPEN ACCESS

Edited by:

Cesare Indiveri,

University of Calabria, Italy

Reviewed by:

Teresa Giraldez,

La Laguna University, Spain Francesco Tadini-Buoninsegni,

University of Florence, Italy

*Correspondence:

Graça Soveral gsoveral@ff.ulisboa.pt

Specialty section:

This article was submitted to Cellular Biochemistry,

a section of the journal

Frontiers in Chemistry

Received: 17 December 2015

Accepted: 12 January 2016

Published: 01 February 2016

Citation:

Madeira A, Moura TF and Soveral G (2016) Detecting Aquaporin Function and Regulation. Front. Chem. 4:3. doi: 10.3389/fchem.2016.00003
Water is the major component of cells and tissues throughout all forms of life. Fluxes of water and solutes through cell membranes and epithelia are essential for osmoregulation and energy homeostasis. Aquaporins are membrane channels expressed in almost every organism and involved in the bidirectional transfer of water and small solutes across cell membranes. Aquaporins have important biological roles and have been implicated in several pathophysiological conditions suggesting a great translational potential in aquaporin-based diagnostics and therapeutics. Detecting aquaporin function is critical for assessing regulation and screening for new activity modulators that can prompt the development of efficient medicines. Appropriate methods for functional analysis comprising suitable cell models and techniques to accurately evaluate water and solute membrane permeability are essential to validate aquaporin function and assess short-term regulation. The present review describes established assays commonly used to assess aquaporin function in cells and tissues, as well as the experimental biophysical strategies required to reveal functional regulation and identify modulators, the first step for aquaporin drug discovery.

Keywords: aquaporin, aquaglyceroporin, membrane, water permeability, glycerol, channel, regulation, inhibition

\section{INTRODUCTION}

Water homeostasis and energy balance are essential for survival and adaptation of living cells. Water crosses cell membranes by two parallel pathways, with distinct mechanisms for permeation: partition/diffusion of water molecules across the hydrophobic bilayer and water diffusion through specialized protein channels known as aquaporins (Verkman, 2000). In either case, lipid or channel pathway, the driving force for water movement is the chemical potential of water (osmotic and/or hydrostatic pressure gradients) between both sides of the membrane. Compared to lipid bilayer diffusion, lower activation energy for transport is a typical feature of aquaporin-mediated diffusion.

Aquaporins (AQPs) belong to a highly conserved group of membrane proteins called the major intrinsic proteins that form a large family comprising more than 1700 integral membrane proteins found in virtually all-living organisms (Abascal et al., 2014). AQPs can be divided into three subfamilies: (i) orthodox or classical aquaporins, considered to be water selective, (ii) aquaglyceroporins, permeable to glycerol and other small solutes in addition to water, and (iii) S-aquaporins, also called unorthodox superaquaporins or subcellular aquaporins, a third subfamily only present in animals but not in plants, fungi and bacteria (Ishibashi et al., 2014) with permeability still uncertain. 
The number of $\mathrm{AQP}$ isoforms expressed varies significantly among organisms. For instance, Escherichia coli possesses one classical AQP (AqpZ) and one AQP-like sequence (glycerol facilitator GlpF; Maurel et al., 1994; Calamita et al., 1995); the yeast Saccharomyces cerevisiae has two orthodox aquaporins (ScAqy1 and ScAqy2) and two aquaglyceroporins (YFL054Cp and ScFps1; Soveral et al., 2010) and plants possess up to 35 different isoforms (Maurel et al., 2008). In mammals 13 isoforms were identified (AQP0-12) and found differentially expressed in organs and tissues involved in fluid absorption or excretion but also in non-fluid transporting tissues like brain, skin, fat, and liver (Ishibashi et al., 2009).

The most remarkable feature of AQP channels is their high selectivity and efficiency on water or glycerol permeation, excluding ions, and protons (Murata et al., 2000). Apart from water and glycerol, a number of other permeants such as urea, ammonia, hydrogen peroxide, carbon dioxide, metalloids, nitric oxide (Wu and Beitz, 2007), and even ions (Yool and Campbell, 2012) were reported to permeate specific AQPs, although the mechanism of permeation is still obscure.

Regulation of AQPs is critical to osmoregulation and water homeostasis in microorganism and in mammalian organs involved in fluid transport (Kortenoeven and Fenton, 2014). Eukaryotic orthodox (water selective) AQPs are frequently regulated post-translationally either by gating, controlling the channels flux rate, or by trafficking, whereby AQPs are shuttled from intracellular storage sites to the plasma membrane (Törnroth-Horsefield et al., 2010). Gating of AQPs has been described for several cell systems. Factors like phosphorylation, $\mathrm{pH}$, pressure, solute gradients, temperature, membrane tension among others, were reported to affect the gating behavior of yeast, plant and mammalian AQPs (Soveral et al., 1997a, 2008; Chaumont et al., 2005; Maurel, 2007; Törnroth-Horsefield et al., 2010; Leitao et al., 2012, 2014; Ozu et al., 2013).

Due to their unique ability to transport glycerol, AQPs play critical roles in osmoregulation by controlling the intracellular accumulation of glycerol. For example, yeast osmostressinduced glycerol accumulation is controlled by the high osmolarity glycerol (HOG) pathway at the level of gene expression, metabolism and transport. Regulation of the yeast aquaglyceroporin Fps1 that changes from open to closed state to ensure intracellular retention and accumulation of glycerol produced by alcohol fermentation is crucial for cells osmoprotective strategy (Ahmadpour et al., 2014).

In mammals, AQPs have also important roles in energy metabolism. By controlling glycerol content in epidermal, fat and other tissues, aquaglyceroporins are involved in skin hydration, cell proliferation, carcinogenesis and fat metabolism (HaraChikuma and Verkman, 2006; Rodríguez et al., 2011; Ribatti et al., 2014). Glycerol permeability in membranes from various tissues and organs has a key role in the regulation of metabolic and energy homeostasis, with the adipose tissue having a pivotal role (Madeira et al., 2015; Rodriguez et al., 2015). Whereas adipose aquaglyceroporin expression is hormonemediated, triggered by catecholamines and insulin in fasting or feeding situations (Fruhbeck et al., 2014), less is known about their short-term regulation or gating.
Additionally in recent years multiple compounds have been described as inhibitors of AQPs water transport activity, but only a limited number was described for glycerol permeation via aquaglyceroporins (de Almeida et al., 2014). AQPs based modulator drugs are predicted to be of broad utility in the treatment of several disorders, such as cerebral edema, cancer, obesity, wound healing, epilepsy, glaucoma, and malaria (de Almeida et al., 2014; Verkman et al., 2014).

This review summarizes the biophysical approaches most frequently used to detect aquaporin activity in tissue and cell membranes and describes the experimental strategies required to uncover functional regulation and screen for chemical modulators.

\section{CELL MODELS FOR FUNCTIONAL ANALYSIS}

Due to the widespread distribution of AQPs in nature, water transport assays have been performed using isolated cells from different organisms, such as bacteria (Delamarche et al., 1999; Mallo and Ashby, 2006), yeast (Soveral et al., 2007; Madeira et al., 2010), and mammalian cells (Solenov et al., 2004; Madeira et al., 2013, 2014a). Intracellular vesicles (Coury et al., 1999; Meyrial et al., 2001) as well as plasma membrane vesicles obtained from animal tissues (mainly kidney or intestinal epithelia) have been used to evaluate AQP activity either in intracellular organelles (Calamita et al., 2005; Noronha et al., 2014) or through epithelial membranes (apical or basolateral; Verkman et al., 1985; Soveral et al., 1997b; Mollajew et al., 2010). Another widely used approach to functionally characterize novel AQP isoforms consists in AQP heterologous expression in Xenopus laevis oocytes, which have very low intrinsic water permeability (Preston et al., 1992). In addition to oocytes, yeast cells lacking endogenous AQPs have been used to detect water transport capacity of mammalian (Pettersson et al., 2006) and plant (Leitao et al., 2012) aquaporins and to develop a generic high-throughput assay to identify functional AQP mutants resistant to freezethawing challenge (To et al., 2015). AQP-transfected cell lines (Ma et al., 1993) and mRNA injected Zebrafish embryos (Ikeda et al., 2011) have also been used for heterologous expression.

AQPs from different organisms have also been purified and reconstituted in liposomes, which allowed establishing their direct role in water/solute transport (van Hoek and Verkman, 1992; Zeidel et al., 1992). Phenotypic analysis of transgenic mice lacking AQPs has also brought new insights into their mechanisms of permeation and revealed their involvement in multiple biological functions, including transepithelial fluid transport, cell migration, brain edema, neuroexcitation, cell proliferation, epidermal water retention, and adipocyte metabolism (Verkman et al., 2014).

\section{PERMEABILITY ASSAYS}

Measurements of water or solute permeability of biological membranes indirectly indicate AQP expression and functional status. Regardless of the biological model used, 
the characterization of $\mathrm{AQP}$ activity and evaluation of water permeability is centered on following cell and/or vesicle volume changes resulting directly from water fluxes driven by osmotic and/or pressure gradients (Figure 1A). On the other hand, for the estimation of solute permeability, both the solute fluxes (driven by solute gradients) and the water fluxes have to be taken into account when analyzing the volume changes (Verkman et al., 2000; Figure 1B). Both the water and the solute fluxes are directly proportional to their respective driving forces, with the proportionality constants being the osmotic permeability $\left(P_{f}\right)$ and the solute permeability $\left(P_{s}\right)$ coefficient, respectively. The rate at which the volume changes occur depends on the fraction of water/or solute that permeates the channel (aqueous pathway) vs. the lipid bilayer diffusion. In addition, evaluating the permeability along temperature allows computing the activation energy $\left(E_{a}\right)$ for transport, a valuable parameter to detect aquaporin functional activity. Water or solute fluxes through a hydrophilic channel pore need lower activation energy $E_{a}$ than fluxes across a hydrophobic lipid bilayer, and thus high permeability and low $E_{a}$ indicate permeation via AQPs. An overview on the equations and parameters used to evaluate membrane water permeability can be found in Soveral et al. (2010).

Techniques to evaluate permeability make use of volumedependent optical properties, such as light transmission (Farinas and Verkman, 1996; Farinas et al., 1997), absorbance (Levin et al., 2007), or scattering (Verkman et al., 1985; Soveral et al., 1997b, 2008) and fluorescence (Hamann et al., 2002; Solenov et al., 2004; Soveral et al., 2007). Equivalent strategies are also commonly used to assess the kinetics of transport in cells and proteoliposomes. Combining different biological models (cells/vesicles/proteoliposomes) and optical detection systems offers many possibilities to conduct research on AQP function. The most commonly used approaches will be further discussed.

\section{Epithelial Assays}

Water permeability may be determined in native epithelial tissues (e.g., intestinal wall, kidney tubules) or in epithelial cell monolayers cultured on permeable supports and mounted on Ussing chambers (Clarke, 2009). In this experimental setting, apical and basolateral membranes of polarized cells face different compartments. By adding a membrane impermeant solute such as sucrose or mannitol to one compartment, the generated transepithelial water flux is measured by the height of the fluid in a capillary tube connected to the other compartment (Dorr et al., 1997). Alternatively, a fluorescent dye is added to the hyperosmotic compartment and the rate of fluorescence change due to dye dilution is used to evaluate the total transepithelial osmotic water permeability (Levin et al., 2006). The total transepithelial permeability is the sum of two pathways, cellular and paracellular, where the presence of AQPs only affects the cellular pathway. The individual membrane permeability from these bipolar epithelial cells can be evaluated using isolated vesicles from either basolateral or apical membranes. This approach is widely used for AQP identification and characterization in epithelial membranes (Verkman et al., 1985; Soveral et al., 1997b; Alves et al., 1999).

\section{Osmotic Swelling Assays}

AQP-mediated water transport can be evaluated by heterologous expression in X. laevis oocytes using an osmotic swelling assay (Preston et al., 1992; Verkman, 2000). Oocytes microinjected with AQP mRNA are subjected to hypo-osmotic gradients and the time course of cell swelling is followed by video microscopy. To test for solute permeability, an inwardly directed solute gradient is imposed, resulting in solute influx in response to the generated chemical gradient, followed by water influx and consequent oocyte swelling (Beitz et al., 2009). This system is particularly advantageous for studying AQPs due to oocytes endogenous low water permeability and hardly detectable glycerol and other solutes permeability.

A similar swelling assay uses erythrocytes expressing endogenous AQPs. Human erythrocytes express only one aquaglyceroporin isoform, AQP3. After challenged with hyperosmotic solute (glycerol) gradients, glycerol influx causes erythrocyte swelling and eventually cell hemolysis that can be monitored as a decrease in light absorption at $625 \mathrm{~nm}$ (Campos et al., 2011). The rate constant of hemolysis can be used to calculate glycerol permeability.

\section{Microscopy Techniques}

Over the years many microscopy techniques have been employed to characterize AQP function, namely phase contrast (Preston et al., 1992), dark field/phase contrast (Farinas et al., 1997), interferometry (Farinas and Verkman, 1996), confocal (Zelenina and Brismar, 2000), and fluorescence microscopy (Verkman, 2000; Solenov et al., 2004; Madeira et al., 2013).

The most currently used microscopy approaches exploit the properties of volume sensitive fluorescent dyes that undergo intracellular de-esterification and trapping. Cells are loaded with the membrane-permeant non-fluorescent precursor (e.g., calcein acetoxymethyl ester), which is cleaved intracellularly by nonspecific esterases yielding the impermeable fluorescent form that gets trapped inside the cells (Madeira et al., 2013). Changes in fluorescence intensity resulting from osmotically induced volume changes can be monitored as the fluorescence of the fluophore is quenched. Two hypotheses explain this phenomenon inside the cell: (1) the quenching is mediated by cytoplasmic proteins, whose concentrations change as cells shrink or swell (Solenov et al., 2004); (2) fluorophores undergo self-quenching, i.e., fluorescence intensity decreases with increasing fluorophore concentration (Hamann et al., 2002). In any case, water and/or solutes permeability can be inferred from the linear relationship between cell volume and dye fluorescence intensity (Hamann et al., 2002).

Another method uses a genetically encoded optical sensor (yellow fluorescent protein YFP-H148Q-V163S), whose fluorescence is quenched by chloride (Galietta et al., 2001; Baumgart et al., 2012). Cell volume changes and consequent altered intracellular chloride concentration results in altered YFP emitted fluorescence.

More recently, a high throughput system for automated water/solute permeability measurements using volume-sensitive fluorescent indicators has been optimized taking advantage of microplate readers (Fenton et al., 2010). 
A

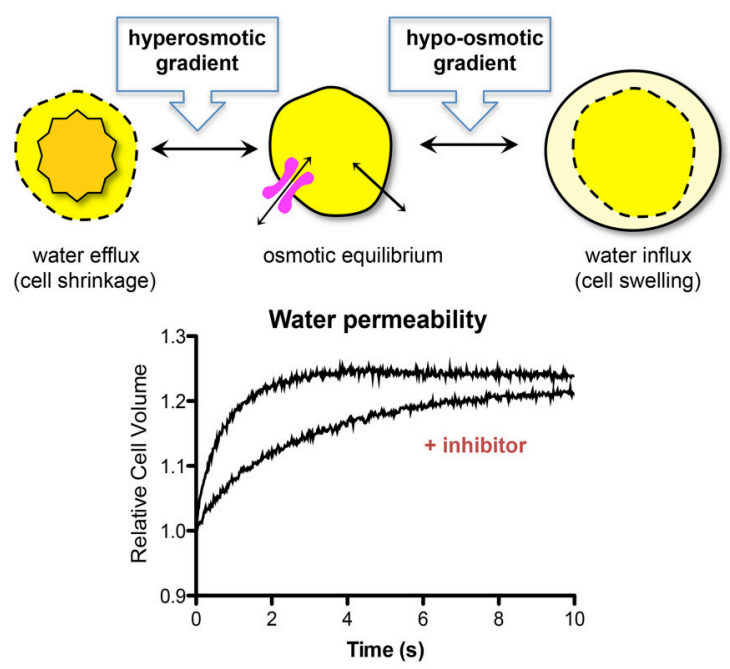

B
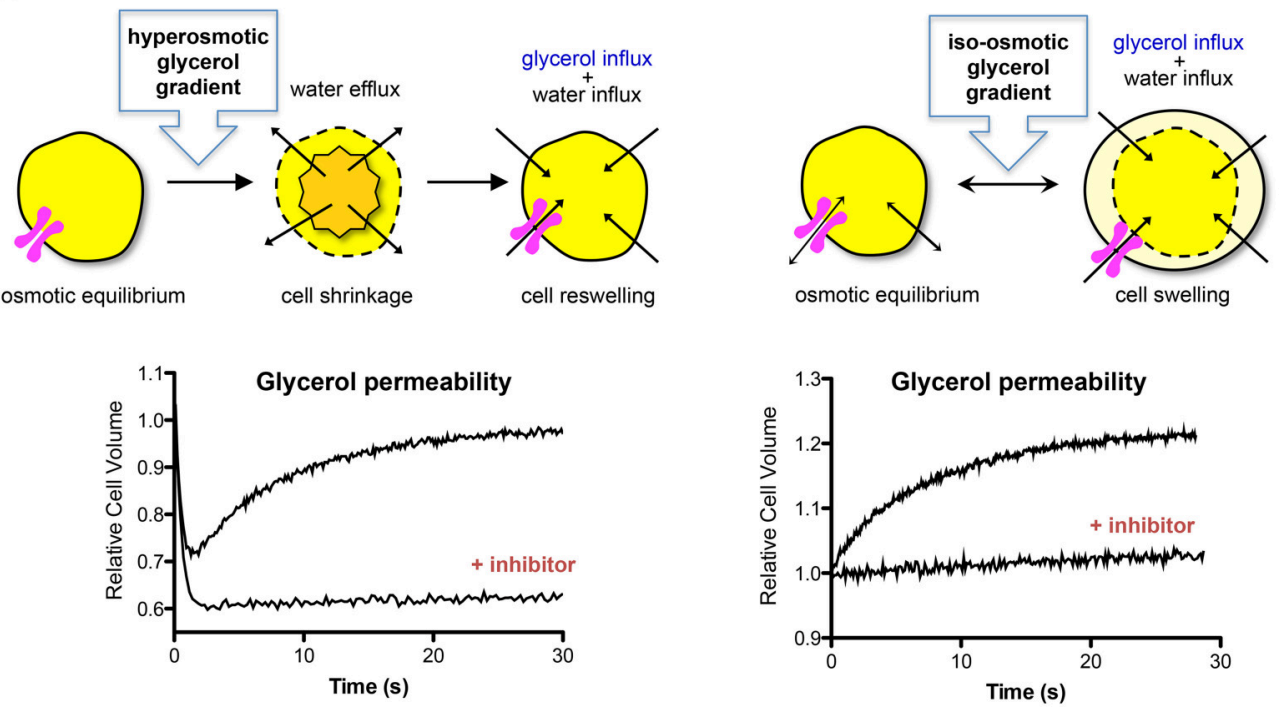

FIGURE 1 | Water and solute transport across biological membranes. (A) Cell volume changes due to water fluxes after an imposed hyperosmotic or hypo-osmotic gradient with an impermeant solute. Water crosses cell membranes simultaneously through the lipid bilayer and AQPs inducing cell shrinkage or swelling, until a new osmotic equilibrium is reached. The presence of functional AQPs increases the rate of cell volume change. The graph shows a typical stopped-flow signal where the rate of volume change after a hypo-osmotic shock induces cell swelling. In the presence of an AQP inhibitor, the rate of swelling is decreased. (B) Cell volume change after imposing a glycerol gradient to cells expressing functional aquaglyceroporins. Left: hyperosmotic glycerol gradient. After the first fast cell shrinkage due to water outflow, glycerol influx in response to its chemical gradient is followed by water influx with subsequent cell reswelling. The graph shows a typical stopped-flow signal where the rate of volume change after imposing a glycerol gradient is biphasic, corresponding to the first fast water efflux followed by glycerol uptake and cell volume recover. In the presence of an aquaglyceroporin inhibitor, the rate of reswelling is strongly reduced. Right: iso-osmotic glycerol gradient. Glycerol influx due to its chemical gradient is followed by water influx with consequent cell swelling. The graph shows a typical stopped-flow signal where the rate of volume change after imposing a glycerol gradient induces cell swelling. An aquaglyceroporin inhibitor drastically reduces the rate of swelling.

\section{Stopped-Flow Spectroscopy}

The stopped flow technique is the leading method to follow the fast-kinetics of cell volume change after a rapidly imposed osmotic/solute gradient. It has been frequently used to measure permeability of cell suspensions (Ma et al., 1993; Dobbs et al., 1998; Soveral et al., 2006, 2007; Martins et al., 2012), vesicles (Verkman et al., 1985; Soveral et al., 1997b; Alves et al., 1999), and proteoliposomes (van Hoek and Verkman, 1992; Zeidel et al., 1992; Noronha et al., 2014).
In the stopped flow device, cell/vesicle suspensions are subjected to osmotic challenges by rapid mixing with an equal volume of hypo- or hyper-osmotic solution (Figure 1). Osmotic water fluxes produce changes in cell volume, with consequent alterations in scattered light intensity or in fluorescence if the cells/vesicles are loaded with volume sensitive fluorescent dyes (e.g., fluorescein or calcein). Due to a linear relation between the optical properties of the system and cell volume, water, or solute movements can be followed until osmotic equilibrium is 
attained. Analysis of the relative volume changes due to osmotic or solute gradients allows quantification of osmotic permeability $\left(P_{f}\right)$ or solute permeability $\left(P_{s}\right)$ coefficients. Figure 1 displays the possible experimental configurations for measuring membrane water and glycerol permeability using the stop-flow technique.

\section{Computational Methods}

The introduction of computational methods has shed new light on AQPs water and solute permeation mechanisms. These methods make use of the available high-resolution atomic structures for different AQP isoforms (Fu et al., 2000; Sui et al., 2001; Savage et al., 2003; Harries et al., 2004; Lee et al., 2005; Törnroth-Horsefield et al., 2006; Horsefield et al., 2008; Newby et al., 2008; Fischer et al., 2009; Ho et al., 2009; Frick et al., 2014). Specifically, molecular dynamics simulations, which employ classical mechanics for the sampling of conformational changes in biomolecules, provided a unique dynamic insight onto AQPs structures (Hub et al., 2009). In the absence of a solved atomic structure for a specific AQP isoform, it is possible to assemble three-dimensional models using experimentally determined structures of related family members as templates (Bordoli et al., 2009). The assembly of homology models, having GlpF channel as a template, allowed accessing the structural uniqueness of human AQP3 (Martins et al., 2012), AQP7 (Madeira et al., 2014b), and AQP9 (Wacker et al., 2013).

\section{ASSESSING AQP INHIBITION AND SHORT-TERM REGULATION}

Short-term regulation of AQPs, also known as gating, is often achieved by mechanisms directly affecting the protein channel conformation, which in turn impacts its transport activity (Alleva et al., 2012). It has been reported gating of eukaryotic AQPs by pH (Nemeth-Cahalan and Hall, 2000; Leitao et al., 2012), phosphorylation (Maurel et al., 1995; Fushimi et al., 1997; Kitchen et al., 2015), divalent cations (Zelenina et al., 2003), and membrane stretching (membrane surface tension; Soveral et al., 1997a, 2008; Ozu et al., 2013; Leitao et al., 2014). A comprehensive review on the therapeutic modulation of aquaporin functionality can be found in Beitz et al. (2015).

$X$. laevis oocytes swelling assays were the first used to show inhibition of AQP1 by mercury chloride (Preston et al., 1992) and are frequently used to detect AQP inhibition (Detmers et al., 2006; Huber et al., 2009; Migliati et al., 2009). However, the preparation procedure of the Xenopus oocytes before permeability assays renders this approach more adequate to validate previous identified inhibitors rather than to screen large libraries of compounds. Disclosure of novel inhibitors was also successfully performed in cultured cell lines selectively expressing AQP isoforms (Gao et al., 2006; Jelen et al., 2011; Wacker et al., 2013; Madeira et al., 2014b).

Control of AQP function by $\mathrm{pH}$ or specific inhibitors, can be screened through simple measurements of permeability and activation energy for water or solute transport after preincubation with the test compound and using the appropriate cell model assay. Human erythrocytes are known to express a large amount of AQP1 and AQP3 accountable for membrane permeability to water and glycerol, respectively (Campos et al., 2011), thus providing a simple screening assay to accurately evaluate inhibition or gating. Using the stopped-flow technique, human erythrocytes have been widely used to disclose AQP inhibitors (Niemietz and Tyerman, 2002; Yang et al., 2006; Martins et al., 2012, 2013). In addition, residues involved in phosphorylation (Maurel et al., 1995; Johansson et al., 1998; Kitchen et al., 2015) and also in any other gating mechanism can be identified in silico and validated in vitro by site-directed mutagenesis.

Levin et al. (2007) developed a simple screening method to identify inhibitors of AQP1 that involves measuring erythrocyte cell lysis using infrared light scattering. Erythrocyte expressing AQP1 and urea transporter (UT-B) were loaded with the urea analog acetamide, in the presence and absence of AQP1 inhibitors. In the absence of inhibitors, dilution of cells in a hypo-osmotic acetamide-free solution resulted in cell swelling and lysis. When AQP1 was inhibited, water influx was slower and dissipation of the osmotic gradient by acetamide efflux prevented cell lysis (Verkman, 2009).

A yeast phenotypic freeze-thaw assay was recently developed to screen for AQP inhibitors. Here, the survival of yeast cells exposed to freezing and thawing is dependent on their membrane water permeability, which is increased by heterologous AQP expression and impaired by inhibitors (To et al., 2015). Although promising as a high-throughput screening system, the identified inhibitors require further validation in a more robust biophysical assay.

Strategies to reveal AQP short-term regulation by membrane tension have been conceived. Membrane tension is related to the force needed to deform a membrane and is directly related to the product of pressure and radius. This means that to reach the same membrane tension, larger cells need much lower levels of pressure than smaller cells, i.e., the larger the cell the lower the pressure needed to trigger membrane deformation. Above a given pressure threshold cell membrane disrupts. Since these minimal pressures are difficult to manipulate experimentally, the effect of membrane tension on AQP activity in large mammalian cells is difficult to detect. Thus, assessment of AQP regulation by tension was achieved using membrane vesicles from mammalian tissues (Soveral et al., 1997a,c), walled yeast and plant cells (Soveral et al., 2008; Leitao et al., 2014), systems that are able to sustain high pressure gradients that induce different levels of tension without membrane rupture. A different approach was implemented by Ozu et al. (2005), using an emptied-out X. laevis oocyte as a diaphragm between two independent chambers. By allowing different levels of hydrostatic pressure, this procedure enabled to study human AQP1 gating by membrane tension (Ozu et al., 2013). Additionally, this system enables controlling the media composition on both compartments and detecting intracellular binding of inhibitors (Ozu et al., 2011).

In silico methods, in particular molecular docking and molecular dynamic simulations have been used to screen large libraries of compounds as candidate AQP inhibitors (Huber et al., 2009; Seeliger et al., 2013; Wacker et al., 2013). These methods are now considered essential for AQP drug discovery providing an educated guess for in vitro testing. 


\section{FINAL REMARKS}

In recent years, our understanding of the pathophysiology of water/glycerol balance disorders has increased enormously. AQPs have been progressively identified as key players in several physiological mechanisms and their dysfunction or aberrant expression implicated in disease, suggesting a great translational potential in aquaporin-based diagnostics and therapeutics.

Detecting AQP function, and characterizing their selectivity and mechanisms of gating is essential to establish AQP contribution to homeostasis, which is critical to health, and further identify dysfunctions that may lead to phenotypes such as those found in disease. In particular, the involvement of aquaglyceroporin-mediated glycerol transport in cell proliferation and adipocyte metabolism and its correlation with metabolic disorders and cancer, unveil the members of the aquaglyceroporin subfamily as promising therapeutic targets.

In spite of the numerous studies already available, several questions still remain open. Future research to untangle the biological relevance of AQPs as cause or consequence of the pathological condition ought to be conducted. Certainly more work is required to validate AQPs as drug targets, including careful analyses of the phenotypes of knockout models as well

\section{REFERENCES}

Abascal, F., Irisarri, I., and Zardoya, R. (2014). Diversity and evolution of membrane intrinsic proteins. Biochim. Biophys. Acta 1840, 1468-1481. doi: 10.1016/j.bbagen.2013.12.001

Ahmadpour, D., Geijer, C., Tamás, M. J., Lindkvist-Petersson, K., and Hohmann, S. (2014). Yeast reveals unexpected roles and regulatory features of aquaporins and aquaglyceroporins. Biochim. Biophys. Acta 1840, 1482-1491. doi: 10.1016/j.bbagen.2013.09.027

Alleva, K., Chara, O., and Amodeo, G. (2012). Aquaporins: another piece in the osmotic puzzle. FEBS Lett. 586, 2991-2999. doi: 10.1016/j.febslet.2012.06.013

Alves, P., Soveral, G., Macey, R. I., and Moura, T. F. (1999). Kinetics of water transport in eel intestinal vesicles. J. Membr. Biol. 171, 177-182. doi: $10.1007 / \mathrm{s} 002329900569$

Baumgart, F., Rossi, A., and Verkman, A. S. (2012). Light inactivation of water transport and protein-protein interactions of aquaporin-Killer Red chimeras. J. Gen. Physiol. 139, 83-91. doi: 10.1085/jgp.201110712

Beitz, E., Becker, D., von Bülow, J., Conrad, C., Fricke, N., Geadkaew, A., et al. (2009). In vitro analysis and modification of aquaporin pore selectivity. Handb. Exp. Pharmacol. 190, 77-92. doi: 10.1007/978-3-540-79885-9_4

Beitz, E., Golldack, A., Rothert, M., and von Bülow, J. (2015). Challenges and achievements in the therapeutic modulation of aquaporin functionality. Pharmacol. Ther. 155, 22-35. doi: 10.1016/j.pharmthera.2015.08.002

Bordoli, L., Kiefer, F., Arnold, K., Benkert, P., Battey, J., and Schwede, T. (2009). Protein structure homology modeling using SWISS-MODEL workspace. Nat. Protoc. 4, 1-13. doi: 10.1038/nprot.2008.197

Calamita, G., Bishai, W. R., Preston, G. M., Guggino, W. B., and Agre, P. (1995). Molecular cloning and characterization of AqpZ, a water channel from Escherichia coli. J. Biol. Chem. 270, 29063-29066. doi: 10.1074/jbc.270.49. 29063

Calamita, G., Ferri, D., Gena, P., Liquori, G. E., Cavalier, A., Thomas, D., et al. (2005). The inner mitochondrial membrane has aquaporin-8 water channels and is highly permeable to water. J. Biol. Chem. 280, 17149-17153. doi: 10.1074/jbc.C400595200

Campos, E., Moura, T. F., Oliva, A., Leandro, P., and Soveral, G. (2011). Lack of Aquaporin 3 in bovine erythrocyte membranes correlates with low glycerol permeation. Biochem. Biophys. Res. Commun. 408, 477-481. doi: 10.1016/j.bbrc.2011.04.057 as pathophysiological studies in humans. In addition, the search for selective and non-toxic inhibitors that could be used as either chemical probes to detect AQP function in biological systems or as innovative therapeutic agents in a variety of disease states, should be encouraged.

These aspects can only be supported if the appropriate methods for functional analysis are conducted, including optimal technical/cell systems and accurate permeability evaluations. The use of in silico models together with well-designed experimental strategies are crucial for assessing AQP function and regulation, noticeably in the pursuit of innovative aquaporin-based drugs.

\section{AUTHOR CONTRIBUTIONS}

AM discussed and wrote the manuscript. TM contributed to discussions and writing; GS proposed the outline, discussed, and supervised the writing.

\section{ACKNOWLEDGMENTS}

This work was supported by Fundação para a Ciência e Tecnologia (PEst-OE/SAU/UI4013/2011-2014 to iMed. ULisboa).

Chaumont, F., Moshelion, M., and Daniels, M. J. (2005). Regulation of plant aquaporin activity. Biol. Cell 97, 749-764. doi: 10.1042/BC20040133

Clarke, L. L. (2009). A guide to Ussing chamber studies of mouse intestine. Am. J. Physiol. Gastrointest. Liver Physiol. 296, G1151-G1166. doi: 10.1152/ajpgi.90649.2008

Coury, L. A., Hiller, M., Mathai, J. C., Jones, E. W., Zeidel, M. L., and Brodsky, J. L. (1999). Water transport across yeast vacuolar and plasma membrane-targeted secretory vesicles occurs by passive diffusion. J. Bacteriol. 181, 4437-4440.

de Almeida, A., Soveral, G., and Casini, A. (2014). Gold compounds as aquaporin inhibitors: new opportunities for therapy and imaging. Med. Chem. Commun. 5, 1444-1453. doi: 10.1039/C4MD00265B

Delamarche, C., Thomas, D., Rolland, J. P., Froger, A., Gouranton, J., Svelto, M., et al. (1999). Visualization of AqpZ-mediated water permeability in Escherichia coli by cryoelectron microscopy. J. Bacteriol. 181, 4193-4197.

Detmers, F. J., de Groot, B. L., Muller, E. M., Hinton, A., Konings, I. B., Sze, M., et al. (2006). Quaternary ammonium compounds as water channel blockers. Specificity, potency, and site of action. J. Biol. Chem. 281, 14207-14214. doi: 10.1074/jbc.M513072200

Dobbs, L. G., Gonzalez, R., Matthay, M. A., Carter, E. P., Allen, L., and Verkman, A. S. (1998). Highly water-permeable type I alveolar epithelial cells confer high water permeability between the airspace and vasculature in rat lung. Proc. Natl. Acad. Sci. U.S.A. 95, 2991-2996. doi: 10.1073/pnas.95.6.2991

Dorr, R. A., Kierbel, A., Vera, J., and Parisi, M. (1997). A new data-acquisition system for the measurement of the net water flux across epithelia. Comput. Methods Programs Biomed. 53, 9-14. doi: 10.1016/S0169-2607(96)01801-9

Farinas, J., Kneen, M., Moore, M., and Verkman, A. S. (1997). Plasma membrane water permeability of cultured cells and epithelia measured by light microscopy with spatial filtering. J. Gen. Physiol. 110, 283-296. doi: 10.1085/jgp.110.3.283

Farinas, J., and Verkman, A. S. (1996). Cell volume and plasma membrane osmotic water permeability in epithelial cell layers measured by interferometry. Biophys. J. 71, 3511-3522. doi: 10.1016/S0006-3495(96)79546-2

Fenton, R. A., Moeller, H. B., Nielsen, S., de Groot, B. L., and Rützler, M. (2010). A plate reader-based method for cell water permeability measurement. Am. J. Physiol. Renal Physiol. 298, F224-F230. doi: 10.1152/ajprenal.00463.2009

Fischer, G., Kosinska-Eriksson, U., Aponte-Santamaria, C., Palmgren, M., Geijer, C., Hedfalk, K., et al. (2009). Crystal structure of a yeast aquaporin at 1.15 angstrom reveals a novel gating mechanism. PLoS Biol. 7:e1000130. doi: 10.1371/journal.pbio. 1000130 
Frick, A., Eriksson, U. K., de Mattia, F., Oberg, F., Hedfalk, K., Neutze, R., et al. (2014). X-ray structure of human aquaporin 2 and its implications for nephrogenic diabetes insipidus and trafficking. Proc. Natl. Acad. Sci. U.S.A. 111, 6305-6310. doi: 10.1073/pnas.1321406111

Frühbeck, G., Méndez-Giménez, L., Fernández-Formoso, J. A., Fernández, S., and Rodríguez, A. (2014). Regulation of adipocyte lipolysis. Nutr. Res. Rev. 27, 63-93. doi: 10.1017/S095442241400002X

Fu, D., Libson, A., Miercke, L. J., Weitzman, C., Nollert, P., Krucinski, J., et al. (2000). Structure of a glycerol-conducting channel and the basis for its selectivity. Science 290, 481-486. doi: 10.1126/science.290. 5491.481

Fushimi, K., Sasaki, S., and Marumo, F. (1997). Phosphorylation of serine 256 is required for CAMP-dependent regulatory exocytosis of the aquaporin-2 water channel. J. Biol. Chem. 272, 14800-14804. doi: 10.1074/jbc.272.23.14800

Galietta, L. J., Haggie, P. M., and Verkman, A. S. (2001). Green fluorescent proteinbased halide indicators with improved chloride and iodide affinities. FEBS Lett. 499, 220-224. doi: 10.1016/S0014-5793(01)02561-3

Gao, J., Wang, X., Chang, Y., Zhang, J., Song, Q., Yu, H., et al. (2006). Acetazolamide inhibits osmotic water permeability by interaction with aquaporin-1. Anal. Biochem. 350, 165-170. doi: 10.1016/j.ab.2006.01.003

Hamann, S., Kiilgaard, J., Litman, T., Alvarez-Leefmans, F., Winther, B., and Zeuthen, T. (2002). Measurement of cell volume changes by fluorescence self-quenching. J. Fluoresc. 12, 139-145. doi: 10.1023/A:1016832027325

Hara-Chikuma, M., and Verkman, A. S. (2006). Physiological roles of glyceroltransporting aquaporins: the aquaglyceroporins. Cell. Mol. Life Sci. 63, 1386-1392. doi: 10.1007/s00018-006-6028-4

Harries, W. E., Akhavan, D., Miercke, L. J., Khademi, S., and Stroud, R. M. (2004). The channel architecture of aquaporin 0 at a 2.2-A resolution. Proc. Natl. Acad. Sci. U.S.A. 101, 14045-14050. doi: 10.1073/pnas.0405274101

Ho, J. D., Yeh, R., Sandstrom, A., Chorny, I., Harries, W. E., Robbins, R. A., et al. (2009). Crystal structure of human aquaporin 4 at $1.8 \mathrm{~A}$ and its mechanism of conductance. Proc. Natl. Acad. Sci. U.S.A. 106, 7437-7442. doi: 10.1073/pnas.0902725106

Horsefield, R., Nordén, K., Fellert, M., Backmark, A., Törnroth-Horsefield, S., Terwisscha van Scheltinga, A. C., et al. (2008). High-resolution x-ray structure of human aquaporin 5. Proc. Natl. Acad. Sci. U.S.A. 105, 13327-13332. doi: $10.1073 /$ pnas.0801466105

Hub, J. S., Grubmüller, H., and de Groot, B. L. (2009). Dynamics and energetics of permeation through aquaporins. What do we learn from molecular dynamics simulations? Handb. Exp. Pharmacol. 190, 57-76. doi: 10.1007/978-3-54079885-9_3

Huber, V. J., Tsujita, M., and Nakada, T. (2009). Identification of aquaporin 4 inhibitors using in vitro and in silico methods. Bioorg. Med. Chem. 17, 411-417. doi: 10.1016/j.bmc.2007.12.040

Ikeda, M., Andoo, A., Shimono, M., Takamatsu, N., Taki, A., Muta, K., et al. (2011). The NPC motif of aquaporin-11, unlike the NPA motif of known aquaporins, is essential for full expression of molecular function. J. Biol. Chem. 286, 3342-3350. doi: 10.1074/jbc.M110.180968

Ishibashi, K., Hara, S., and Kondo, S. (2009). Aquaporin water channels in mammals. Clin. Exp. Nephrol. 13, 107-117. doi: 10.1007/s10157-008-0118-6

Ishibashi, K., Tanaka, Y., and Morishita, Y. (2014). The role of mammalian superaquaporins inside the cell. Biochim. Biophys. Acta 1840, 1507-1512. doi: 10.1016/j.bbagen.2013.10.039

Jelen, S., Wacker, S., Aponte-Santamaria, C., Skott, M., Rojek, A., Johanson, U., et al. (2011). Aquaporin-9 protein is the primary route of hepatocyte glycerol uptake for glycerol gluconeogenesis in mice. J. Biol. Chem. 286, 44319-44325. doi: 10.1074/jbc.M111.297002

Johansson, I., Karlsson, M., Shukla, V. K., Chrispeels, M. J., Larsson, C., and Kjellbom, P. (1998). Water transport activity of the plasma membrane aquaporin PM28A is regulated by phosphorylation. Plant Cell 10, 451-459. doi: 10.1105/tpc.10.3.451

Kitchen, P., Öberg, F., Sjöhamn, J., Hedfalk, K., Bill, R. M., Conner, A. C., et al. (2015). Plasma membrane abundance of human aquaporin 5 is dynamically regulated by multiple pathways. PLOS ONE 10:e0143027. doi: 10.1371/journal.pone.0143027

Kortenoeven, M. L., and Fenton, R. A. (2014). Renal aquaporins and water balance disorders. Biochim. Biophys. Acta 1840, 1533-1549. doi: 10.1016/j.bbagen.2013.12.002
Lee, J. K., Kozono, D., Remis, J., Kitagawa, Y., Agre, P., and Stroud, R. M. (2005) Structural basis for conductance by the archaeal aquaporin AqpM at $1.68 \mathrm{~A}$ Proc. Natl. Acad. Sci. U.S.A. 102, 18932-18937. doi: 10.1073/pnas.0509469102

Leitao, L., Prista, C., Loureiro-Dias, M. C., Moura, T. F., and Soveral, G. (2014). The grapevine tonoplast aquaporin TIP2;1 is a pressure gated water channel. Biochem. Biophys. Res. Commun. 450, 289-294. doi: 10.1016/j.bbrc.2014.05.121

Leitao, L., Prista, C., Moura, T. F., Loureiro-Dias, M. C., and Soveral, G. (2012). Grapevine aquaporins: gating of a tonoplast intrinsic protein (TIP2;1) by cytosolic pH. PLoS ONE 7:e33219. doi: 10.1371/journal.pone.0033219

Levin, M. H., de la Fuente, R., and Verkman, A. S. (2007). Urearetics: a small molecule screen yields nanomolar potency inhibitors of urea transporter UT-B. FASEB J. 21, 551-563. doi: 10.1096/fj.06-6979com

Levin, M. H., Sullivan, S., Nielson, D., Yang, B., Finkbeiner, W. E., and Verkman, A. S. (2006). Hypertonic saline therapy in cystic fibrosis: evidence against the proposed mechanism involving aquaporins. J. Biol. Chem. 281, 25803-25812. doi: 10.1074/jbc.M604332200

Ma, T., Frigeri, A., Tsai, S. T., Verbavatz, J. M., and Verkman, A. S. (1993). Localization and functional analysis of CHIP28k water channels in stably transfected Chinese hamster ovary cells. J. Biol. Chem. 268, 22756-22764.

Madeira, A., Camps, M., Zorzano, A., Moura, T. F., and Soveral, G. (2013). Biophysical assessment of human aquaporin-7 as a water and glycerol channel in 3T3-L1 adipocytes. PLoS ONE 8:e83442. doi: 10.1371/journal.pone.0083442

Madeira, A., de Almeida, A., de Graaf, C., Camps, M., Zorzano, A., Moura T. F., et al. (2014b). A gold coordination compound as a chemical probe to unravel aquaporin-7 function. Chembiochem 15, 1487-1494. doi: 10.1002/cbic. 201402103

Madeira, A., Fernandez-Veledo, S., Camps, M., Zorzano, A., Moura, T. F. Ceperuelo-Mallafre, V., et al. (2014a). Human aquaporin-11 is a water and glycerol channel and localizes in the vicinity of lipid droplets in human adipocytes. Obesity 22, 2010-2017. doi: 10.1002/oby.20792

Madeira, A., Leitao, L., Soveral, G., Dias, P., Prista, C., Moura, T., et al. (2010). Effect of ethanol on fluxes of water and protons across the plasma membrane of Saccharomyces cerevisiae. FEMS Yeast Res. 10, 252-258. doi: 10.1111/j.15671364.2010.00607.x

Madeira, A., Moura, T. F., and Soveral, G. (2015). Aquaglyceroporins: implications in adipose biology and obesity. Cell. Mol. Life Sci. 72, 759-771. doi: 10.1007/s00018-014-1773-2

Mallo, R. C., and Ashby, M. T. (2006). AqpZ-mediated water permeability in Escherichia coli measured by stopped-flow spectroscopy. J. Bacteriol. 188, 820-822. doi: 10.1128/JB.188.2.820-822.2006

Martins, A. P., Ciancetta, A., de Almeida, A., Marrone, A., Re, N., Soveral, G., et al. (2013). Aquaporin inhibition by gold(III) compounds: new insights. ChemMedChem 8, 1086-1092. doi: 10.1002/cmdc.201300107

Martins, A. P., Marrone, A., Ciancetta, A., Galan Cobo, A., Echevarria, M., Moura, T. F., et al. (2012). Targeting aquaporin function: potent inhibition of aquaglyceroporin-3 by a gold-based compound. PLOS ONE 7:e37435. doi: 10.1371/journal.pone.0037435

Maurel, C. (2007). Plant aquaporins: novel functions and regulation properties. FEBS Lett. 581, 2227-2236. doi: 10.1016/j.febslet.2007.03.021

Maurel, C., Kado, R. T., Guern, J., and Chrispeels, M. J. (1995). Phosphorylation regulates the water channel activity of the seed-specific aquaporin alpha-TIP. EMBO J. 14, 3028-3035.

Maurel, C., Reizer, J., Schroeder, J. I., Chrispeels, M. J., and Saier, M. H. Jr. (1994). Functional characterization of the Escherichia coli glycerol facilitator, GlpF, in Xenopus oocytes. J. Biol. Chem. 269, 11869-11872.

Maurel, C., Verdoucq, L., Luu, D. T., and Santoni, V. (2008). Plant aquaporins: membrane channels with multiple integrated functions. Annu. Rev. Plant Biol. 59, 595-624. doi: 10.1146/annurev.arplant.59.032607.092734

Meyrial, V., Laize, V., Gobin, R., Ripoche, P., Hohmann, S., and Tacnet, F. (2001) Existence of a tightly regulated water channel in Saccharomyces cerevisiae. Eur. J. Biochem. 268, 334-343. doi: 10.1046/j.1432-1033.2001.01882.x

Migliati, E., Meurice, N., DuBois, P., Fang, J. S., Somasekharan, S., Beckett, E., et al. (2009). Inhibition of aquaporin-1 and aquaporin- 4 water permeability by a derivative of the loop diuretic bumetanide acting at an internal pore-occluding binding site. Mol. Pharmacol. 76, 105-112. doi: 10.1124/mol.108.053744

Mollajew, R., Zocher, F., Horner, A., Wiesner, B., Klussmann, E., and Pohl, P. (2010). Routes of epithelial water flow: aquaporins versus cotransporters. Biophys. J. 99, 3647-3656. doi: 10.1016/j.bpj.2010.10.021 
Murata, K., Mitsuoka, K., Hirai, T., Walz, T., Agre, P., Heymann, J. B., et al. (2000). Structural determinants of water permeation through aquaporin-1. Nature 407, 599-605. doi: 10.1038/35036519

Nemeth-Cahalan, K. L., and Hall, J. E. (2000). pH and calcium regulate the water permeability of aquaporin 0. J. Biol. Chem. 275, 6777-6782. doi: $10.1074 /$ jbc. 275.10 .6777

Newby, Z. E., O’Connell, J. III, Robles-Colmenares, Y., Khademi, S., Miercke, L. J., and Stroud, R. M. (2008). Crystal structure of the aquaglyceroporin PfAQP from the malarial parasite Plasmodium falciparum. Nat. Struct. Mol. Biol. 15, 619-625. doi: 10.1038/nsmb.1431

Niemietz, C. M., and Tyerman, S. D. (2002). New potent inhibitors of aquaporins: silver and gold compounds inhibit aquaporins of plant and human origin. FEBS Lett. 531, 443-447. doi: 10.1016/S0014-5793(02)03581-0

Noronha, H., Agasse, A., Martins, A. P., Berny, M. C., Gomes, D., Zarrouk, O., et al. (2014). The grape aquaporin VvSIP1 transports water across the ER membrane. J. Exp. Bot. 65, 981-993. doi: 10.1093/jxb/ert448

Ozu, M., Dorr, R., and Parisi, M. (2005). New method to measure water permeability in emptied-out Xenopus oocytes controlling conditions on both sides of the membrane. J. Biochem. Biophys. Methods 63, 187-200. doi: 10.1016/j.jbbm.2005.04.007

Ozu, M., Dorr, R. A., Gutierrez, F., Politi, M. T., and Toriano, R. (2013). Human AQP1 is a constitutively open channel that closes by a membrane-tensionmediated mechanism. Biophys. J. 104, 85-95. doi: 10.1016/j.bpj.2012.11.3818

Ozu, M., Dorr, R. A., Teresa Politi, M., Parisi, M., and Toriano, R. (2011). Water flux through human aquaporin 1: inhibition by intracellular furosemide and maximal response with high osmotic gradients. Eur. Biophys. J. 40, 737-746. doi: 10.1007/s00249-011-0687-2

Pettersson, N., Hagstrom, J., Bill, R. M., and Hohmann, S. (2006). Expression of heterologous aquaporins for functional analysis in Saccharomyces cerevisiae. Curr. Genet. 50, 247-255. doi: 10.1007/s00294-006-0092-z

Preston, G. M., Carroll, T. P., Guggino, W. B., and Agre, P. (1992). Appearance of water channels in Xenopus oocytes expressing red cell CHIP28 protein. Science 256, 385-387. doi: 10.1126/science.256.5055.385

Ribatti, D., Ranieri, G., Annese, T., and Nico, B. (2014). Aquaporins in cancer. Biochim. Biophys. Acta 1840, 1550-1553. doi: 10.1016/j.bbagen.2013.09.025

Rodríguez, A., Catalán V, Gómez-Ambrosi, J., and Frühbeck, G. (2011). Aquaglyceroporins serve as metabolic gateways in adiposity and insulin resistance control. Cell Cycle 10, 1548-1556. doi: 10.4161/cc.10.10.15672

Rodriguez, A., Ezquerro, S., Mendez-Gimenez, L., Becerril, S., and Frühbeck, G. (2015). Revisiting the adipocyte: a model for integration of cytokine signaling in the regulation of energy metabolism. Am. J. Physiol. Endocrinol. Metabol. 309, E691-E714. doi: 10.1152/ajpendo.00297.2015

Savage, D. F., Egea, P. F., Robles-Colmenares, Y., O'Connell, J. D. III, and Stroud, R. M. (2003). Architecture and selectivity in aquaporins: 2.5 a X-ray structure of aquaporin Z. PLoS Biol. 1:E72. doi: 10.1371/journal.pbio.0000072

Seeliger, D., Zapater, C., Krenc, D., Haddoub, R., Flitsch, S., Beitz, E., et al. (2013). Discovery of novel human aquaporin-1 blockers. ACS Chem. Biol. 8, 249-256. doi: $10.1021 / \mathrm{cb} 300153 \mathrm{z}$

Solenov, E., Watanabe, H., Manley, G. T., and Verkman, A. S. (2004). Sevenfoldreduced osmotic water permeability in primary astrocyte cultures from AQP-4deficient mice, measured by a fluorescence quenching method. Am. J. Physiol. Cell Physiol. 286, C426-C432. doi: 10.1152/ajpcell.00298.2003

Soveral, G., Macey, R. I., and Moura, T. F. (1997a). Membrane stress causes inhibition of water channels in brush border membrane vesicles from kidney proximal tubule. Biol. Cell 89, 275-282. doi: 10.1111/j.1768322X.1997.tb01023.x

Soveral, G., Macey, R. I., and Moura, T. F. (1997b). Water permeability of brush border membrane vesicles from kidney proximal tubule. J. Membr. Biol. 158, 219-228. doi: 10.1007/s002329900259

Soveral, G., Macey, R. I., and Moura, T. F. (1997c). Mechanical properties of brush border membrane vesicles from kidney proximal tubule. J. Membr. Biol. 158, 209-217. doi: 10.1007/s002329900258

Soveral, G., Madeira, A., Loureiro-Dias, M. C., and Moura, T. F. (2007). Water transport in intact yeast cells as assessed by fluorescence self-quenching. Appl. Environ. Microbiol. 73, 2341-2343. doi: 10.1128/AEM.02519-06

Soveral, G., Madeira, A., Loureiro-Dias, M. C., and Moura, T. F. (2008). Membrane tension regulates water transport in yeast. Biochim. Biophys. Acta 1778, 2573-2579. doi: 10.1016/j.bbamem.2008.07.018
Soveral, G., Prista, C., Moura, T. F., and Loureiro-Dias, M. C. (2010). Yeast water channels: an overview of orthodox aquaporins. Biol. Cell 103, 35-54. doi: 10.1042/BC20100102

Soveral, G., Veiga, A., Loureiro-Dias, M. C., Tanghe, A., Van Dijck, P., and Moura, T. F. (2006). Water channels are important for osmotic adjustments of yeast cells at low temperature. Microbiology 152(Pt 5), 1515-1521. doi: 10.1099/mic.0.28679-0

Sui, H., Han, B. G., Lee, J. K., Walian, P., and Jap, B. K. (2001). Structural basis of water-specific transport through the AQP1 water channel. Nature 414, 872-878. doi: $10.1038 / 414872 \mathrm{a}$

To, J., Yeo, C. Y., Soon, C. H., and Torres, J. (2015). A generic highthroughput assay to detect aquaporin functional mutants: potential application to discovery of aquaporin inhibitors. Biochim. Biophys. Acta 1850, 1869-1876. doi: 10.1016/j.bbagen.2015.05.019

Törnroth-Horsefield, S., Hedfalk, K., Fischer, G., Lindkvist-Petersson, K., and Neutze, R. (2010). Structural insights into eukaryotic aquaporin regulation. FEBS Lett. 584, 2580-2588. doi: 10.1016/j.febslet.2010.04.037

Törnroth-Horsefield, S., Wang, Y., Hedfalk, K., Johanson, U., Karlsson, M., Tajkhorshid, E., et al. (2006). Structural mechanism of plant aquaporin gating. Nature 439, 688-694. doi: 10.1038/nature04316

van Hoek, A. N., and Verkman, A. S. (1992). Functional reconstitution of the isolated erythrocyte water channel CHIP28. J. Biol. Chem. 267, 18267-18269.

Verkman, A. S. (2000). Water permeability measurement in living cells and complex tissues. J. Membr. Biol. 173, 73-87. doi: 10.1007/s002320001009

Verkman, A. S. (2009). Aquaporins: translating bench research to human disease. J. Exp. Biol. 212(Pt 11), 1707-1715. doi: 10.1242/jeb.024125

Verkman, A. S., Anderson, M. O., and Papadopoulos, M. C. (2014). Aquaporins: important but elusive drug targets. Nat. Rev. Drug Discov. 13, 259-277. doi: $10.1038 / \mathrm{nrd} 4226$

Verkman, A. S., Dix, J. A., and Seifter, J. L. (1985). Water and urea transport in renal microvillus membrane vesicles. Am. J. Physiol. 248(5 Pt 2), F650-F655.

Verkman, A. S., Yang, B., Song, Y., Manley, G. T., and Ma, T. (2000). Role of water channels in fluid transport studied by phenotype analysis of aquaporin knockout mice. Exp. Physiol. 85, Spec. No:233S-41S. doi: 10.1111/j.1469445X.2000.tb00028.x

Wacker, S. J., Aponte-Santamaría, C., Kjellbom, P., Nielsen, S., de Groot, B. L., and Rützler, M. (2013). The identification of novel, high affinity AQP9 inhibitors in an intracellular binding site. Mol. Membr. Biol. 30, 246-260. doi: 10.3109/09687688.2013.773095

Wu, B., and Beitz, E. (2007). Aquaporins with selectivity for unconventional permeants. Cell. Mol. Life Sci. 64, 2413-2421. doi: 10.1007/s00018-007-7163-2

Yang, B., Kim, J. K., and Verkman, A. S. (2006). Comparative efficacy of $\mathrm{HgCl} 2$ with candidate aquaporin-1 inhibitors DMSO, gold, TEA+ and acetazolamide. FEBS Lett. 580, 6679-6684. doi: 10.1016/j.febslet.2006.11.025

Yool, A. J., and Campbell, E. M. (2012). Structure, function and translational relevance of aquaporin dual water and ion channels. Mol. Aspects Med. 33, 553-561. doi: 10.1016/j.mam.2012.02.001

Zeidel, M. L., Ambudkar, S. V., Smith, B. L., and Agre, P. (1992). Reconstitution of functional water channels in liposomes containing purified red cell CHIP28 protein. Biochemistry 31, 7436-7440. doi: 10.1021/bi00148a002

Zelenina, M., Bondar, A. A., Zelenin, S., and Aperia, A. (2003). Nickel and extracellular acidification inhibit the water permeability of human aquaporin-3 in lung epithelial cells. J. Biol. Chem. 278, 30037-30043. doi: 10.1074/jbc.M302206200

Zelenina, M., and Brismar, H. (2000). Osmotic water permeability measurements using confocal laser scanning microscopy. Eur. Biophys. J. 29, 165-171. doi: 10.1007/PL00006645

Conflict of Interest Statement: The authors declare that the research was conducted in the absence of any commercial or financial relationships that could be construed as a potential conflict of interest.

Copyright (c) 2016 Madeira, Moura and Soveral. This is an open-access article distributed under the terms of the Creative Commons Attribution License (CC BY). The use, distribution or reproduction in other forums is permitted, provided the original author(s) or licensor are credited and that the original publication in this journal is cited, in accordance with accepted academic practice. No use, distribution or reproduction is permitted which does not comply with these terms. 